\title{
Ribelli dello Spirito tra estremo oriente ed estremo occidente
}

\author{
Francesca Rachele Oppedisano ${ }^{1}$
}

In the 1960s, at the height of Japan's cultural reformation by a Western-style liberal democratic constitution, the Japanese writer Yukio Mishima (Tokyo, 1925-1970) decided to lay down the weapons of literature to personify the hero of Japan's cultural restoration, opposing the country's gradual colonization by Western civilization with the ancient world of the samurai warrior. In this last period of his life Mishima prepared his individual reaction, rebelling against the Word and its discursive regimes as being responsible for a radical misunderstanding of the individual, who is constantly surpassed by something that speaks and legislates in his stead. In 1970 Mishima completed his task by stabbing himself in the stomach in the ancient samurai tradition. Mishima was one of the finest examples of a rebellious spirit.

Durante gli Anni Sessanta del secolo scorso, all'apice dello sviluppo della cultura giapponese riformata secondo i canoni liberaldemocratici di stampo occidentale, lo scrittore giapponese Yukio Mishima (Tokyo 1925 - 1970) decide di deporre le armi della poesia e della letteratura per incarnare l'eroe della restaurazione. Contro la progressiva colonizzazione del mondo orientale da parte dell'occidente, Mishima oppone l'antico mondo dei guerrieri e dei samurai ribellandosi alla parola e ai suoi regimi discorsivi in quanto responsabili del radicale fraintendimento del soggetto, costantemente ecceduto da qualcosa che discorre e, soprattutto, legifera al suo posto. Nel 1970 Mishima porterà a termine il suo compito infliggendosi un taglio nello stomaco alla maniera dei samurai. Resta tra gli esempi più rimarchevoli di ribelli dello spirito.

\section{Yukio Mishima. Il tramonto dell'Oriente tra nichilismo e volontà di potenza}

«Se Dio non esistesse più, bisognerebbe farlo rinascere $»^{2}$.

Yukio Mishima crede che un Dio sia ancora necessario per plasmare un individuo capace di sorpassare le minacciose insidie del nichilismo e della trascendenza e che al contempo, attraverso un estremo atto vitale, sia in grado di raggiungere l'Assoluto attraverso la morte. Tra estremo occidente nietzschiano ed estremo oriente buddhista, all'interno di questa dicotomia, tra volontà

\footnotetext{
${ }^{1}$ Storica dell'arte, Ufficio Mostre, Palazzo delle Esposizioni di Roma.

${ }^{2}$ Cf. il colloquio tra Mishima e il critico letterario Furubayashi Takashi avvenuto nel 1970 in Takashi FURUBAYASHI, Hideo KOBAYASHI, Le ultime parole di Mishima, (dir.) Emanuele CICCARELLA, Milano, Universale Economica Feltrinelli, 2001, p. 36.
} 
di potenza e radicale annientamento del tutto, si situa questa particolare figura d'artista ${ }^{3}$. Ci troviamo all'interno di un dualismo, ci dice lo stesso Mishima in una intervista del 1970 che cade a pochi mesi dalla morte ${ }^{4}$ che l'Oriente non concepisce e che si appropria della sua visione del mondo a cominciare dalla lettura de La Nascita della Tragedia di Friedrich Nietzsche. Apollineo e Dionisiaco trovano in Mishima il loro corrispettivo nella via della penna e della spada, nei cosiddetti fiumi dell'azione e della prosa. Una coppia di opposti destinata a ricongiungersi, sarebbe meglio dire a reincarnarsi, come vedremo, nel recupero tardivo di una figura mitica, quella del Samurai.

Sì, sognare di raggiungere l'Assoluto. Sognare, sognare, ma poiché questo sogno è romantico non può essere realizzato. L'impossibilità di tale realizzazione è l'arte, mentre la sua possibilità è l'azione. Questa è la via della penna e della spada. Il raggiungimento dell'Assoluto,... è la morte. Per l'arte l'dea della morte non è praticabile. L'arte deve vivere, vivere a lungo... Ma se parliamo di azione, allora si può anche morire a diciotto anni. Solo allora si raggiunge la perfezione [...] Tutto ciò mi ha fatto pensare che dovevo prendere una decisione come artista 5 .

Ciò che muove l'arte è sempre qualcosa di essenziale e sempre identico, ciò che muove la vita di ciascun individuo è qualcosa di unico e irripetibile. Questo dualismo informa la sua arte e il suo modo di concepire la sua opera secondo una struttura che egli stesso definisce ad archi : co-

\footnotetext{
${ }^{3}$ Yukio MISHIMA, pseudonimo di Kimitake Hiraoka, nasce a Tokio nel 1925 e muore suicida nel 1970. La sua prima opera letteraria, un racconto in prima persona marcatamente autobiografico dal titolo Confessioni di una Maschera (Kamen no kokuhaku), appare nel 1949 e vende circa ventimila copie dando l'avvio al suo successo come scrittore. Non si tratta di un romanzo dell'io, la prima persona narrante è, per così dire, in maschera e mantiene rispetto all'io narrato un distacco prossimale. Si tratta di fatto di una sorta di auto analisi nella sua fase rielaborativa, quando il ricordo e la ripetizione hanno lasciato spazio alla verità, è necessario precisare, del racconto. La vasta eco che ebbe il romanzo in Oriente e in Occidente può essere dovuta, sul piano contenutistico, alla formazione letteraria di Mishima : nell'aver portato in essere temi appartenenti alla letteratura occidentale (Dostoevskij, Wilde, Baudelaire, Proust, Cocteau, Radiguet) all'interno di un racconto riferito ad una educazione radicalmente tradizionalista. L'opera sarà pubblicata in italiano, tradotta dall'inglese, nel 1964 e nel 1969 direttamente dal giapponese. Già prima di allora e durante l'intero arco della sua vita letteraria, suo mentore e fedele sostenitore è lo scrittore giapponese Kawabata Yasunari (1899-1972), vincitore del Nobel per la letteratura nel 1968.

${ }^{4}$ Ivi, p. 67

${ }^{5}$ Ivi, p. 69.
} 
me un arco necessita dell'altra metà per sorreggersi la sua arte richiede due forze contrapposte che ne determinino e ne garantiscano l'equilibrio. Nel mantenimento di questa struttura statica e dinamica al tempo stesso Mishima intende superare qualsiasi relativismo :

" poiché viviamo in questo mondo di blando relativismo, paradossalmente, per affermare l'Assoluto è meglio accettare un forte dualismo o estremo relativismo " ${ }^{6}$. Al tempo stesso bisogna sottolineare che Mishima non rinnega la tradizione spirituale giapponese e la visione del mondo che la caratterizza : il Giappone non contempla ambivalenze, tutto è ridotto ad una cosa sola, sintetizza sempre Mishima nell'intervista sopraccitata ${ }^{7}$. Il pensatore giapponese Tsujimura Kōichi osserva che a partire dall'europeizzazione del Giappone in epoca Meiji, processo acuitosi a partire dal secondo dopoguerra, il pensiero giapponese ha dovuto necessariamente confrontarsi con la cultura europea : « onde poter conservare la nostra indipendenza nel mondo attuale, vale a dire nell'ambito di potenza [Machtbereich] determinato dalla volontà. Ma, nel contempo » poiché questo confronto non è avvenuto attraverso un processo di rielaborazione della cultura nipponica « in essa vi è il pericolo di smarrire la nostra peculiare essenza $»^{8}$. Il Giappone, di fatto, soffre di una scissione tra la propria visione della natura e « la maniera occidentale di vivere e pensare, determinata dalla volontà ${ }^{9}$ che lo costringe, a partire dal quotidiano, a vivere una doppia vita. A nostro avviso è a cominciare da queste essenziali osservazioni filosofiche, nel mantenimento inconciliabile di queste due prospettive sul mondo, che si può tentare una visione critica di un artista che ha sperimentato su di sé la radicale, drammatica impossibilità di elaborare un incontro autentico tra oriente e occidente. Al di là dei pericoli dettati dalla resa storica, politica e filosofica a oriente, oltre i confini della volontà di potenza affidata ad un pensiero che proprio in quegli anni a occidente comincia a perdere terreno a favore di una volontà di conoscenza affidata all' automatismo della tecnica, si situano molto spesso degli spiriti eroici, molto particolari, la cui morte nulla ha a che fare con il sacrificio, ma piuttosto con il tentativo di riconciliare, diremmo noi occidentali, pensiero e vita, Mishima avrebbe detto spirito e natura.

\footnotetext{
${ }_{7}^{6} I v i$, pp. 67-68.

${ }^{7}$ Ivi, p. 67.

${ }^{8}$ Tsujimura ha trattato le corrispondenze tra il pensiero di Martin Heidegger e il Buddhismo zen. Koichi TSUJIMURA, Martin Heideggers Denken und die japanische Philosophie. Festrede zum 26. September 1969, in Ansprachen zum 80. Geburtstag am 26. September 1969 in Meßkirch (hg. von der Stadt Meßkirch), Meßkirch 1970, pp. 9-19 ; tr. it. di Carlo SAVIANI, Martin Heidegger e la filosofia giapponese, discorso per l'ottantesimo compleanno di Heidegger tenuto da Tsujimura Koichi, ora in www.asia.it

${ }^{9}$ Ibidem.
} 


\section{Al di là del mondo come volontà e rappresentazione}

Nel suo secondo romanzo (Colori proibiti, 1951) Mishima lascia dire ad uno dei protagonisti le seguenti parole : « La questione davvero fondamentale è se sia possibile o no la contemporaneità di espressione e azione $»^{10}$. Il buddhismo zen, come la filosofia di Heidegger, ci ricorda Tsujimura Koichi oltrepassano la rappresentazione soggettiva del mondo - « stendono al suolo il pensiero rappresentativo $\gg-{ }^{11}$ ponendosi entro una circolarità che elude qualsiasi rapporto soggetto e oggetto ${ }^{12}$.

Si tratta, su un piano filosofico, di operare ad Occidente una piccola morte del soggetto e a Oriente « un epocale pensiero e messa in questione del mondo $»^{13}$.

$\mathrm{Al}$ di là della rappresentazione Mishima pone un io originario, autentico e adolescenziale, che cade prima di qualsiasi artificio o finzione. Bisogna fare molta attenzione perché il termine rappresentazione, che tanto intimamente si lega a finzione (quindi alla ripetizione e mai all'assoluto), appartiene alla visione del mondo di Mishima per reazione, la causa è ravvisabile in un incidente, remoto, che lui stesso definì "la prima vendetta della verità", a cui reagì attraverso la costruzione dei suoi padiglioni fatti di parole. In tenera età, di fronte alla riproduzione di un giovane guerriero che alimentava le sue fantasie, la domestica di casa gli domandò se conoscesse la storia dello sventurato guerriero :

« Sembra un uomo, non è vero ? In realtà è una donna ; è la storia di una donna che si finge uomo...»

«Una donna?»

Ero annichilito. La persona che credevo un uomo era in realtà una donna. Cosa accade se un bel cavaliere non è un uomo ma una donna ? [...] Sembrava la prima vendetta della verità con cui mi sarei scontrato nella mia vita, una vendetta particolarmente crudele verso le dolci

\footnotetext{
${ }^{10}$ Y. MISHIMA, « Colori proibiti », tr. it. di Maria Gioia VIENNA, in Id., Romanzi e racconti, (dir. e intr.) Maria Teresa ORSI, Milano, Mondadori, vol. I, 1949-1961, p. 815.

${ }^{11}$ K. TSUJIMURA, Discorso per l'ottantesimo compleanno di Heidegger, op. cit., www.asia.it

${ }^{12}$ Ibidem. « Il buddhismo zen caratterizza questa situazione, ad esempio, in questo modo : L'asino guarda nel pozzo e il pozzo nell'asino. L'uccello guarda il fiore e il fiore guarda l'uccello ».

${ }^{13}$ Ibidem.
} 
fantasie che nutrivo sulla sua morte... Da quel giorno riposi il libro illustrato e non lo ripresi più $^{14}$.

Così come una immagine può celare un significato diverso dal suo apparire che, lo ricordiamo, in Giappone non si offre come possibilità altra, poiché le cose sono come sono, anche la parola viene avvertita da Mishima come un ostacolo.

Se in un primo momento Mishima recupera dalla tradizione culturale del suo paese l'idea di un potere arcaico e magico della parola, più tardi, nel saggio intitolato Sole e Acciaio (1968) ne sottolinea il potere corrosivo e riconosce al corpo la vera potenza di ricongiungimento con il tutto : «Quando mi sono reso conto che l'esercizio della forza, la fatica che ne conseguiva, il sudore, le lacrime e il sangue potevano rilevare ai miei occhi il sacro, immutabile cielo azzurro [...] ho avuto il presentimento, forse, che in un giorno lontano avrei potuto superare i limiti della mia individualità nei quali le parole mi avevano forzato $»^{15}$. In Lezioni spirituali per giovani Samurai afferma : "soltanto in anni recenti ho capito che bastava praticare il kendo e brandire una spada di bambù per evadere, anche solo per brevi istanti, dal nichilismo ${ }^{16}$, poiché le parole sono divenute false « come la plastica che imita il marmo $\|^{17}$.

Il corpo diventa dunque viatico per trascendere i limiti della propria conformazione s-oggetiva, frutto di contingenze familiari, culturali e religiose. Dove non può la parola subentra il corpo che siamo : l'esercizio dei muscoli svolge una funzione tonica non solo dal punto di vista fisico ma anche spirituale ${ }^{18}$. Il corpo è anche il viatico per divenire puro spirito, o meglio, pura volontà :

«Anche i muscoli avevano cessato di esistere. Ero immerso in una sensazione di potere come in una luce trasparente $\gg{ }^{19}$ una luce prossima a quello stato di estasi che segue il supplizio dei santi, come nella rappresentazione assai nota del San Sebastiano di Guido Reni che trafitto dalle frecce volge lo sguardo alla grazia di un dolore mutato in beatitudine :

\footnotetext{
${ }^{14}$ Y. MISHIMA, « Confessioni di una maschera » (1949), in Id., Romanzi e racconti, op. cit., Vol I, p. 73. Corsivo nostro.

${ }^{15}$ Y. MISHIMA Yukio, Sole e acciaio, tr.it. di Adrian POPA, Milano, Edizione Borghese, 1972, cit. in Maria Teresa ORSI, «La neve e il sangue », in Y. MISHIMA, Romanzi e racconti , op. cit., p. XIX.

${ }^{16}$ Y. MISHIMA, Lezioni spirituali per giovani samurai, tr.it. di Lydia ORIGLIA, Milano, Feltrinelli, 1990, p. 51.

${ }^{17}$ Ivi, p. 62

${ }^{18}$ Y. MISHIMA, Sole e acciaio, cit. in Maria Teresa ORSI, « La neve e il sangue », op. cit., p. XIX.

${ }^{19}$ Cf. su questi temi : Y. MISHIMA, Sole e acciaio (1970), Milano, Guanda, 1982.
} 
Prima di quel giorno non avevo mai visto né tenuto in mano un libro d'arte... Ne aprii uno verso la fine, e in un angolo di una pagina sulla sinistra apparve un ritratto che sembrava fosse lì per me, che mi stesse aspettando con ansia. Si trattava del San Sebastiano di Guido Reni conservato a Palazzo Rosso, a Genova... Nell'attimo in cui vidi il quadro la mia esistenza venne sconvolta da una gioia pagana : il mio sangue iniziò a scorrere all'impazzata e i miei organi si tinsero del colore dell'ira ${ }^{20}$.

Ma è anche e soprattutto l'estasi del samurai nel momento in cui si dà la morte tramite il rituale del seppuku ${ }^{21}$. La solitudine del guerriero si traduce in elevazione spirituale attraverso la sofferenza corporale, associandosi così alle sofferenze dello spirito. Un rituale solitario che con la definitiva perdita di senso a causa della restaurazione Meiji acquista la bellezza di un gesto gratuito. Il seppuku è un atto simbolico quanto definitivo, ma allo stesso tempo, Mishima sembra sapere che una volta abitata la scissione in cui si ritrova il Giappone modernizzato, la solitudine, intesa come raccoglimento e recupero di una posizione autentica del mondo ad Oriente, per essere compresa in tutto il suo valore simbolico necessita di testimoni, deve passare attraverso la rappresentazione, deve diventare un atto pubblico, come vedremo.

\section{II Nulla Assoluto}

Mishima afferma che l'essenza dell'arte consiste nel « poter ripetere un evento decisivo... È dunque finzione $»^{22}$. È nemica dell'Assoluto in quanto ripetibile eternamente. Per questo i samurai «disdegnavano le rappresentazioni teatrali, ad eccezione del No, le cui regole impongono una sola recita... un'unicità simile a quella di una azione reale..., la bellezza dell'azione è ineluttabilmente legata all'impossibilità di una ripetizione $»^{23}$. Così i protagonisti della tetralogia intito-

\footnotetext{
${ }^{20}$ Y. MISHIMA, Confessioni di una maschera, op. cit., pp. 90-92.

${ }^{21}$ Il seppuku rientra nel codice morale del guerriero e non ha altra funzione che ribadire le proprie virtù. È una pratica solitaria che in nulla incide sull'assetto sociale. Il seppuku fu leggendariamente attribuito al brigante Hakamadare Yasusuke che si tolse la vita al momento della sua cattura dalle forze dell'ordine nel 988 a.c. nasce come atto di rivendicazione del singolo di fronte all'ordine costituito, un atto di irrimediabile e incorreggibile dissenso.

${ }^{22}$ Y. MISHIMA, «Introduzione alla filosofia dell'azione », in Id., Lezioni spirituali per giovani samurai, op. cit., p. 96.

${ }^{23}$ Ibidem.
} 
lata Il mare della fertilità (1965-1970) ${ }^{24}$ « vivono una vita assoluta, irripetibile. Tutto alla fine si fonde nel grande relativismo della filosofia yuishik, tutto entra nel nirvana $»^{25}$ si estingue nell'ultimo stadio della vita ascetica in cui si raggiunge il Nulla. Nota la Marguerite Yourcenar nel suo saggio dedicato a Mishima interrogandosi sulla visione spirituale dell'autore : « ciò che alla fine risulta è Niente, il Nulla, resta da sapere se questo niente, che si avvicina forse al Nada dei mistici spagnoli, coincida, completamente con quello che chiamiamo in francese rien $»^{26}$. La domanda della Yourcenar ci costringe, con Heidegger non a caso, a riformulare la domanda fondamentale di ogni domandare filosofico : «Perché è in generale l'ente e non piuttosto il Niente? ${ }^{27}$. Al di là della logica, la domanda fondamentale viene all'essere a cominciare da uno stato d'animo, ontologicamente rivelativo. Nello "spaesamento dell'angoscia", nella sospensione che essa provoca, l'ente nel suo insieme è privato del suo fondamento. "Ciò implica che noi stessi, questi esseri umani che siamo, in mezzo all'ente ci sentiamo dileguare con esso $»^{28}$ Se l'angoscia « rivela il Niente » allo stesso tempo « ci mozza la parola ${ }^{29}$, ci priva della possibilità di dire : $e^{30}$.

Ma, l'Essere e il Niente, ci dice Heidegger, sono la stessa $\operatorname{cosa}^{31}$ e non è un caso se questa identità venga subito compresa nella verità degli insegnamenti zen. Nella contemplazione ogni azione è destinata al vuoto : « in un contemplare, che per sé sfugge a ogni percezione visiva, il quale si fissa con tanta concentrazione nel vuoto che in questo e per virtù di questo la montagna appare. Il vuoto è allora lo stesso che il Nulla $»^{32}$. Il Nulla non è inteso nihilisticamente, per il Giappone « il Vuoto è il nome più alto» per mostrare l'Essere inteso come Verità.

\footnotetext{
${ }^{24}$ Y.MISHIMA, Il mare della fertilità : Neve di primavera, tr. it di Andrea Maurizi, A briglia sciolta, tr. it. di Lorenzo COSTANTINI, Il Tempio dell'alba e La decomposizione dell'angelo, tr. it. di Emanuele CICCARELLA, in Id, Romanzi e Racconti, op. cit., pp. 209-1774

${ }^{25}$ T. FURUBAYASHI, H. KOBAYASHI, Le ultime parole di Mishima, op. cit., p. 69.

${ }^{26}$ Marguerite YOURCENAR, Mishima o La visione del Vuoto, tr. it. di Laura GUARINO, Milano, Bompiani, 1982, p. 81.

${ }^{27}$ M. HEIDEGGER, Che cosa è Metafisica ?, (dir. e tr.) Franco VOLPI, Milano, Adelphi Edizioni, 2001, p. 67.

${ }^{28}$ Ivi, p. 51.

${ }^{29}$ Ibidem.

${ }^{30}$ M. HEIDEGGER, Che cosa è Metafisica ?, op.cit. Corsivo nostro.

${ }^{31}$ «Se l'esserci, nel fondo della sua essenza, non trascendesse, ossia, come ora possiamo dire, non si tenesse immerso fin dall'inizio nel Niente, non potrebbe mai rapportarsi all'ente, e perciò neanche a se stesso ». Martin HEIDEGGER, Che cosa è Metafisica ?, op. cit., p. 55. Cf. inoltre relativa nota.

${ }^{32} \mathrm{M}$. HEIDEGGER, « Da un colloquio nell'ascolto del linguaggio », in Id, In cammino verso il linguaggio, (dir.) Alberto CARACCIOLO, tr.it. di Alberto CARACCIOLO e Maria CARACCIOLO PERNOTTI, Milano, Mursia
} 
È in questa impossibilità che si gioca anche la verità della parola, nelle aporie cui essa allude a partire dall'erranza del senso rispetto a ciò che da sempre si intende significare. Nel punto di arresto si apre la partita con la morte e con quel Niente che, è importante chiarirlo, non rappresenta l'origine né l'ambizione ultima del senso. Lo zen, osserva Roland Barthes « tout entier mène la guerre contre la prévarication du sens. On sait que le bouddhisme dé joue la voie fatale de toute assertation (ou de toute négation) [...] Lorsque le Sixième Patriarchie donne ses instructions concernant le mondo [...] il recommande [...] dès un terme est posé, de se déporter vers son terme averse ('Si, vous questionnant, quelqu'un vous interroge sur l'être, répondez par le non-être $[\ldots])\rangle^{33}$.

\section{II coraggio di morire. Fare il Vuoto}

« Se incontri il Buddha uccidilo ; se incontri i genitori, uccidili ; se incontri i parenti, uccidili ! Soltanto così potrai ottenere la salvazione dell'anima tua $»^{34}$. Un comandamento Zen che, nota la Yourcenar, è di « una saggezza rischiosa, ma vivificante, di un fervore più libero e di un assoluto mortalmente puro $»^{35}$. Ma, secondo la Yourcenar, le forme del distacco, dell'impermanenza e del Vuoto assumono per Mishima sempre maggiore importanza, fatta eccezione per la pietas.

«Mishima si è voluto spietato $»^{36}$. Di una spietata coerenza. Nel 1961 pubblica un racconto dal titolo Patriottismo ${ }^{37}$ da cui nel 1965 trarrà un mediometraggio ${ }^{38}$. Come spesso in Mishima il racconto è tratto da un fatto realmente accaduto, il cosiddetto incidente del 26 febbraio1936 : un gruppo di militari, guidati da giovani ufficiali, tentano un colpo di stato, stroncato due giorni dopo. Due degli ufficiali si uccidono. È il racconto degli ultimi momenti del giovane tenente e della sua giovane moglie morti suicidi tramite il rituale del seppuku. In un articolo apparso nel 1966 (L'incidente del 26 febbraio ed io) Mishima scrive : « Il tenente e la moglie, in una circostanza

Editore, 1973, p. 97. In questo colloquio gli interlocutori sono un Giapponese (il Prof. Tezuka dell'Università Imperiale di Tokio) e un Interrogante (lo stesso Heidegger), come premesso alla trascrizione del dialogo, avvenuta in seguito a questo incontro, tra il 1953 e il 1954.

${ }^{33}$ Roland BARTHES, « L'exemption du sens », in id., L'empire des signes, Paris, Editions du Seuil, 2005.

${ }^{34}$ Citato in Marguerite YOURCENAR, Mishima o La visione del Vuoto, op. cit., p. 31.

${ }^{35}$ Ibidem.

${ }^{36}$ M. YOURCENAR, Mishima o La visione del Vuoto, op. cit,, pp. $42-43$ e nota.

${ }^{37}$ Y. MISHIMA, « Patriottismo » (1961), tr. it. di Michela MORRESI, in Id., Romanzi e racconti, op. cit., vol. I, pp. 1669-1698.

${ }^{38}$ Cortometraggio del 1965 diretto e interpretato dallo stesso Mishima. 
tragica, hanno afferrato senza saperlo il momento supremo dell'esistenza e sono morti di una morte che è la massima beatitudine $»{ }^{39}$. Ripensando a questo episodio e al suo racconto Mishima si riferisce esplicitamente a Nietzsche e a George Bataille. I temi che riguardano l'eros e la morte in vista del seppuku sono motivati dall'idea portante che la bellezza possa essere raggiunta solo e unicamente attraverso un evento unico e irripetibile (a sventare l'eterno ritorno) e sono mossi dalla certezza della fine come ciò che conferisce alle cose transeunti e relative della vita l'Unicità dell'Assoluto. La consapevolezza della fine garantisce il massimo dell'erotismo la cui bellezza si configura come necessaria perché esistente per l'ultima volta. Così questo atto consumato in solitudine non somiglia ad una sconfitta perché, ad un grado più elevato, si tratta di una battaglia dello spirito : " In quel campo di battaglia non ci sarebbe stata gloria, nessuno avrebbe potuto dimostrare il proprio valore, era la linea del fronte dell'anima $»^{40}$.

L'ultima battaglia sul fronte dell'anima Mishima l'avrebbe consumata il 25 novembre del 1970.

\section{Volere il Nulla piuttosto che non volere}

Per affrontare il capitolo che segue è necessario una breve digressione nelle regioni del pensiero filosofico nietzschiano che tanto hanno influenzato il nostro artista. Enucleandone in estrema sintesi il cardine sintetizzato dal concetto di volontà di potenza. La volontà di potenza è « il tratto fondamentale di ogni ente », nel costante «trapasso di qualcosa a qualcos'altro ${ }^{41}$. Essa è il divenire « delle condizioni di conservazione-accrescimento in ordine alle formazioni complesse di relativa durata in seno alla vita $»^{42}$. «Le complesse formazioni della vita dipendono da condizioni di conservazione tali che il permanente sussiste soltanto per il farsi in-permanente nell'accrescimento ${ }^{43}$. In seno al divenire, la vita, cioè il vivente, si concentra in vari centri della volontà di potenza. Essi sono l'arte, lo Stato, la religione, la scienza, la società ${ }^{44}$. «Volere è in sé in se stesso comandare », ma non comandare ad un altro bensì il comandare della volontà a se

\footnotetext{
${ }^{39}$ Cf. Y. MISHIMA, Romanzi e Racconti, Milano, Mondadori, vol. I, Notizie sui testi, « Patriottismo », p. 1740.

${ }^{40}$ Ibidem.

${ }^{41}$ M. HEIDEGGER , «La sentenza di Nietzsche : 'Dio è morto' », in Id., Sentieri interrotti, op. cit.. p. 209.

${ }^{42}$ Friedrich NIETZSCHE, Volontà di potenza, Af 715, citato da Heidegger in Sentieri interrotti, p. 208.

${ }^{43} \mathrm{Ivi}$, p. 210.

${ }^{44}$ Ivi, p. 211.
} 
stessa $^{45}$. Ciò che è voluto dalla volontà non è una mancanza. Essa non vuole altro che il suo volere... La volontà vuole se stessa ${ }^{46}$. «Essa [la volontà] vuole il nulla piuttosto che non volere» ${ }^{47}$. Volere il nulla significa non contrapporsi al reale ma « volere il reale come nullità e, quindi, attraverso questa, volere l'annullamento $»^{48}$.

\section{L'ultima battaglia sul fronte dell'anima}

L'ultimo periodo della vita di Mishima, l'ultimo quindicennio che lo separa dalla morte, è stato caratterizzato da un cambio progressivo di postura. Da scrittore affermato, riconosciuto prontamente dall'Occidente come suo prossimo (anche se dal suo punto di vista nutre seri dubbi sul fatto di esser stato compreso fino in fondo) ${ }^{49}$ Mishima, mosso da un deciso anti americanismo e completamente dedito alla restaurazione di un Giappone pre-modernizzato, si rivela sostenitore della destra nipponica. Vede negli ideali che muovono i movimenti di lotta studentesca del '68 un limite insito nel tentativo di trasportare ideali occidentali nella cultura giapponese, ma soprattutto non vi scorge lo spirito autentico che muove i veri guerrieri : « un uomo deve essere in grado di liberarsi da questo attaccamento alla vita e avere il coraggio di morire $»^{50}$. La vita, afferma, è solo un aspetto relativo dell'esistenza. Di qui l'avversione per il sistema repubblicano frutto di idee relativistiche, poiché « se non ci sono comandamenti e proibizioni non si può raggiungere l'Assoluto $»^{51}$. È come se Mishima avesse acuito una forza spirituale radicalizzandola in spinta politica : « Lo spiritualismo grezzo e cupo mi piace. Amo il fanatismo, l'oscurantismo, rappresentano il Dioniso che è dentro di me $»^{52}$. Così l'Imperatore, non quello vero, modernizzato, privato dei suoi poteri divini, assurge a figura simbolica, quasi provocatoria, volta a riaccendere la

\footnotetext{
${ }^{45}$ Ivi, p. 214.

${ }^{46} \mathrm{Ivi}$, p. 215.

${ }^{47}$ F. NIETZSCHE, Genealogia della Morale, Sezione III, Af. I, anno 1887, citato da Heidegger in Sentieri interrotti, op. cit., p. 216.

49 « ho sempre avuto l'impressione che gli occidentali si limitassero ad accarezzarmi la testa come si fa con i bambini, pensando con un certo stupore : 'Ma guarda! Per appartenere ad un popolo estremo-orientale così remoto, scrive cose piuttosto interessanti !' ». Cf. Y. MISHIMA, Lezioni spirituali per giovani samurai, op. cit., p.16.

${ }^{50}$ Y. MISHIMA, Le ultime parole di Mishima, op.cit. p. 52.

${ }^{51}$ Ivi, p. 53.

${ }^{52} \mathrm{Ivi}$, p. 83 e sgg.
} 
fiamma « dello spirito dei guerrieri che nel Giappone modernizzato si sta estinguendo $»^{53}$. Mosso da un rinnovato spirito samurai, lo scrittore fonda nel 1968 una associazione paramilitare, il Tatenokai, L'Associazione degli Scudi, un esercito privato composto da giovani studenti universitari. Nel novembre del 1970 Mishima è curatore e redattore del catalogo di una mostra autobiografica. Lettere - Teatro - Corpo - Azione, sono le quattro sezioni in cui si divide la mostra, quattro fiumi ideali che hanno caratterizzato la vita dello scrittore ${ }^{54}$. La mostra espone una sua gigantografia nelle vesti del San Sebastiano di Guido Reni. Ritorna il tema del martirio e dell'estasi che fu il suo principio e la sua fine. Il mattino del 25 novembre 1970 pone fine a I sette segni di decomposizione dell'angelo, ultimo capitolo della tetralogia intitolata Il mare della fertilità, lascia il manoscritto nell'ingresso di casa. Insieme ai quattro più fidati membri del $\mathrm{Ta}$ tenokai, si reca presso la sede dell'esercito di autodifesa giapponese. Con un atto di forza occupa l'ufficio del generale Mashita. Dal balcone dell'ufficio, di fronte a un migliaio di uomini del reggimento di fanteria, oltre che a giornali e televisioni, tiene il suo ultimo discorso :

Siamo furibondi per il troppo lungo sonno in cui giace il Giappone del dopoguerra !... Abbiamo creduto che il Giappone si sarebbe destato soltanto quando l'esercito avesse riaperto gli occhi... Quattro anni orsono, io, animato da un simile proposito, mi arruolai nell'esercito, e l'anno seguente costituii l'Associazione degli Scudi...il principio fondamentale dell'esercito giapponese non può essere altro che : "proteggere la storia, la cultura, le tradizioni del Giappone fondate sul suo Imperatore."... Abbiamo atteso quattro anni... non possiamo più attendere... Attenderemo ancora solo trenta minuti, gli ultimi trenta minuti insorgeremo insieme e insieme moriremo per l'onore... Avete tanto cara la vita da sacrificarle l'esistenza dello spirito ?... Noi ora testimonieremo a tutti voi l'esistenza di un valore più alto del rispetto della vita. Questo valore non è la libertà, non è la democrazia. È il Giappone... Abbiamo intrapreso questa azione nell'ardente speranza che voi tutti, a cui è stato donato un animo purissimo, possiate ritornare ad essere veri uomini, veri guerrieri ${ }^{55}$.

\footnotetext{
${ }^{53}$ Ivi, p. 65 .

${ }^{54}$ Per la ricostruzione in particolare degli ultimi anni di vita dello scrittore Cf. Henry SCOTT STOKES, Vita e morte di Yukio Mishima, tr. it. di Riccardo MAINARDI, Torino, Lindau, 2008.

${ }^{55}$ Y. MISHIMA, discorso pronunciato pochi attimi prima di morire il 25 novembre 1970. Cf. Y. Mishima, « Proclama », in Lezioni Spirituali per giovani samurai, op. cit., pp.125-126.
} 
Osserva la Yourcenar : " questo scrittore che ha constatato l'insipidezza delle parole crede forse che l'eloquenza avrà maggior potere ? $\nu^{56}$. A conclusione della proclama Mishima rientra nell'ufficio del generale e si toglie la vita assieme al suo compagno di lotta prediletto, Masakatsu Morita.

Due teste sul tappeto sicuramente in acrilico dell'ufficio del generale, messe una accanto all'altra come birilli [...] due teste, due bocche inerti, due cervelli... Due teste mozzate... che a guardarle suscitano sbigottimento più che terrore. Ogni giudizio di valore, sia esso morale, politico o estetico, in loro presenza, momentaneamente almeno, è ridotto al silenzio. Fra le miriadi di cose che sono, e che sono state, queste due teste sono state; e sono [...] Ciò che riempie quegli occhi senza sguardo non è più lo sventolante vessillo della protesta... Due oggetti, relitti già quasi inorganici di annientate strutture, che anch'essi, una volta passati attraverso il fuoco, saranno ridotti a residui minerali e cenere ; [...] Due relitti, sospinti dal Fiume dell'Azione, e che l'immensa ondata ha lasciato per un attimo in secca sulla sabbia, e poi trascina via ${ }^{57}$.

Poco prima di uscire da casa, quella mattina, Mishima aveva redatto diverse lettere destinate ad amici, parenti e giornalisti e soprattutto ad uno dei quattro membri dell'Associazione degli Scudi che sarebbero sopravvissuti all'azione. Chiede di vestire il proprio cadavere in uniforme, quell'uniforme disegnata da lui stesso sul modello delle divise di De Gaulle. Chiede ai genitori che il suo nome postumo, come da tradizione buddhista, contenga l'ideogramma $\mathrm{Bu}$, guerriero. Ma tutto ciò era già stato annunciato da tempo. Nel 1951, nel romanzo Colori proibiti, scriveva :

A causa della bellezza lo spirito non conosce momenti di pace [...] a questo mondo ci sono quelli che definiamo supremi [...] sono gli istanti della riconciliazione tra spirito e natura [...] La loro espressione non si può che definire impossibile fintanto che l'essere umano è vivo [...] L'uomo non può esprimere l'istante supremo in cui diviene tale. L'Artista non è onnipotente [...] Espressione o azione ? [...] non c'è dubbio che nella morte possa esistere contemporaneità tra l'azione che chiamiamo suicidio e l'espressione totale della vita [...] Il

\footnotetext{
${ }^{56}$ M. YOURCENAR, Mishima o La visione del vuoto, op. cit., p. 104.

${ }^{57}$ Ivi, pp. 110-111.
} 
6a Francesca Oppedisano: Ribelli dello Spirito tra estremo oriente ed estremo occidente

punto più alto dell'espressione dell'uomo vivo è qualcosa situato immediatamente dopo l'istante supremo, è la forma totale della vita meno $\alpha^{58}$.

Mishima Yukio a nostro avviso dovrebbe essere ripensato ricominciando da qui. È stato sicuramente ciò che hanno percepito oriente e occidente in superficie, un pensatore pericoloso, una figura pubblica provocatoria e sconcertante, uno scrittore tra i più occidentali e allo stesso tempo tra i più anacronisticamente orientali del novecento, ma al fondo, ciò che più conta e sempre si dimentica, è stato un artista di una estrema lealtà, se l'opera d'arte, come noi crediamo, viene intesa come promessa di vita, Mishima è tra i pochi che hanno saputo mantenere la parola data, in questo consiste il senso profondo del suo rilancio finale.

\footnotetext{
${ }^{58}$ Y. MISHIMA, Colori proibiti, in Id, Romanzi e Racconti, op. cit., vol. I, p. 816.
} 